\title{
Pressure-induced concomitant topological and metal-insulator quantum phase transitions in $\mathrm{Ce}_{3} \mathrm{Pd}_{3} \mathrm{Bi}_{4}$
}

\author{
Chenchao Xu $\mathbb{D}^{1,2,5}$, Chao Cao $\mathbb{i D}^{1,3 凶}$ and Jian-Xin Zhu $\mathbb{i D}^{4 凶}$
}

The electronic property and magnetic susceptibility of $\mathrm{Ce}_{3} \mathrm{Pd}_{3} \mathrm{Bi}_{4}$ were systemically investigated from 18 to $290 \mathrm{~K}$ for varying values of cell volume using dynamic mean-field theory coupled with density functional theory. By extrapolating to zero temperature, the ground state of $\mathrm{Ce}_{3} \mathrm{Pd}_{3} \mathrm{Bi}_{4}$ at ambient pressure is found to be a correlated semimetal due to insufficient hybridization. Upon applying pressure, the hybridization strength increases and a crossover to the Kondo insulator is observed at finite temperatures. The characteristic temperature signaling the formation of Kondo singlet, as well as the characteristic temperature associated with $f$ electron delocalization-localization change, simultaneously vanishes around a critical volume of $0.992 V_{0}$, suggesting that such metal-insulator transition is possibly associated with a quantum critical point. Finally, Wilson's loop calculations indicate that the Kondo insulating side is topologically trivial, thus a topological transition also occurs across the quantum critical point.

npj Quantum Materials (2022)7:18; https://doi.org/10.1038/s41535-022-00427-9

\section{INTRODUCTION}

Kondo insulator is a prototypical strongly correlated quantum matter involving $4 f$ or $5 f$ electrons. At higher temperatures, the system is metallic since the $f$-electron-derived local moments do not strongly couple to conduction electrons. Upon cooling, an energy gap opens at the Fermi energy, due to the formation of Kondo singlets between the local moments and conduction electrons. In the meantime, the electronic states experience an incoherent to coherent crossover. In the above process, the hybridization strength $\Gamma \propto N_{0}|V|^{2}$, with $V$ being the hybridization interaction and $N_{0}$ conduction-electron density of states at the Fermi energy, is of particular importance, since the Kondo coupling strength depends directly on this quantity as $J_{K} \propto|V|^{2} / U$ with $U$ the Coulomb interaction on $f$-electrons. According to the famous Doniach phase diagram ${ }^{1}$, this coupling leads to a conduction-electron mediated Ruderman-Kittel-Kasuya-Yosida (RKKY) interaction, which in turns competes with the Kondo coherence effect. They are characterized by respective energy scale, $T_{\text {RKKY }}$ and $T_{\mathrm{K}}$. Therefore, if $V$ is too small, the formation of Kondo singlet shall fail and lead to a magnetic ground state instead. Apart from temperature, the strength of hybridization can be tuned via chemical doping, external pressure and magnetic field alternatively. These nonthermal parameters therefore provide control over competing ground states, and may realize quantum critical phenomena ${ }^{2-4}$. More interestingly, when the competing ground states are associated with different topological invariants, a topological quantum phase transition is achieved. It has been proposed that the critical point of a topological quantum phase transition can host novel semimetal states, which exhibit nonFermi liquid or marginal Fermi-liquid behavior ${ }^{5-8}$.

The archetypal Kondo insulator $\mathrm{Ce}_{3} \mathrm{Pt}_{3} \mathrm{Bi}_{4}$ was comprehensively and systematically studied in the past several decades ${ }^{9-13}$, and regained much attention recently due to its possible connection with topological Kondo insulators ${ }^{14-16}$; while an isoelectronic substitution of $5 d$-element $\mathrm{Pt}$ with $4 d$ element $\mathrm{Pd}$, the possible nontrivial topological properties and the metallic ground state with a large quasiparticle mass enhancement have been studied in $\mathrm{Ce}_{3} \mathrm{Pd}_{3} \mathrm{Bi}_{4}{ }^{17-20}$. Further experiments, however, show that $\mathrm{Ce}_{3} \mathrm{Pd}_{3} \mathrm{Bi}_{4}$ exhibits a weak activation behavior of $\Delta \sim 0.4 \mathrm{meV}$ which can be suppressed above the magnetic field of $B_{\mathrm{c}} \approx 11 \mathrm{~T}$, suggesting that the system may be very close to a metal-insulator transition point ${ }^{21}$. Theoretically, the dynamical mean-field theory (DMFT) study revealed that the $\mathrm{Ce}_{3} \mathrm{Pd}_{3} \mathrm{Bi}_{4}$ compound is a topological nodal-line semimetal, whose metallic behavior persists down to $4 \mathrm{~K}$, while the $\mathrm{Ce}_{3} \mathrm{Pt}_{3} \mathrm{Bi}_{4}$ compound is a trivial Kondo insulator with an indirect gap of $6 \mathrm{meV}$ below $18 \mathrm{~K}^{19}$. A sharp difference between the hybridization functions of $\mathrm{Ce}_{3} \mathrm{Pt}_{3} \mathrm{Bi}_{4}$ and $\mathrm{Ce}_{3} \mathrm{Pd}_{3} \mathrm{Bi}_{4}$ is observed in the DFT + DMFT study ${ }^{19}$, and in DFT study as well ${ }^{22}$. Nevertheless, it has yet to be determined whether the ground state of $\mathrm{Ce}_{3} \mathrm{Pd}_{3} \mathrm{Bi}_{4}$ compound is a small gap insulator or an exotic metal. Although the ground state study of this compound is of significant importance, an accurate DMFT calculation at sufficiently low temperature is extremely timeconsuming and unrealistic. Here, we take an alternative approach by performing a series of calculations at different temperatures and pressure. As a result, the magnitude of the hybridization energy gap, hybridization strength, valence band edge $E_{\mathrm{v}}$ quasiparticle weight $Z$, as well as local magnetic susceptibility can be studied as functions of temperature and pressure. In addition, the ground state properties can also be obtained by extrapolating temperature-dependent electronic and magnetic properties of $\mathrm{Ce}_{3} \mathrm{Pd}_{3} \mathrm{Bi}_{4}$ to zero temperature.

\section{RESULTS}

Electronic structure of $\mathbf{C e}_{3} \mathbf{P d}_{3} \mathbf{B i}_{4}$

The momentum-resolved spectra functions $A(\omega, \mathbf{k})$ between $E_{\mathrm{F}}-$ $0.3 \mathrm{eV}$ and $E_{\mathrm{F}}+0.2 \mathrm{eV}$ at $T=29,58$, and $116 \mathrm{~K}$ for $90 \% V_{0}, 94 \% V_{0}$,

\footnotetext{
'Department of Physics and Center for Correlated Matter, Zhejiang University, 310058 Hangzhou, P. R. China. ${ }^{2}$ Department of Applied Physics, University of Tokyo, Tokyo 113 8656, Japan. ${ }^{3}$ Condensed Matter Group, School of Physics, Hangzhou Normal University, 311121 Hangzhou, P. R. China. ${ }^{4}$ Theoretical Division and Center for Integrated Nanotechnologies, Los Alamos National Laboratory, Los Alamos, NM 87545, USA. ${ }^{5}$ Present address: Center for Green Research on Energy and Environmental Materials (GREEN) and International Center for Materials Nanoarchitectonics (MANA), National Institute for Materials Science (NIMS), Tsukuba, Ibaraki 305-0044, Japan. ${ }^{\circ}$ email: ccao@zju.edu.cn; jxzhu@lanl.gov
} 


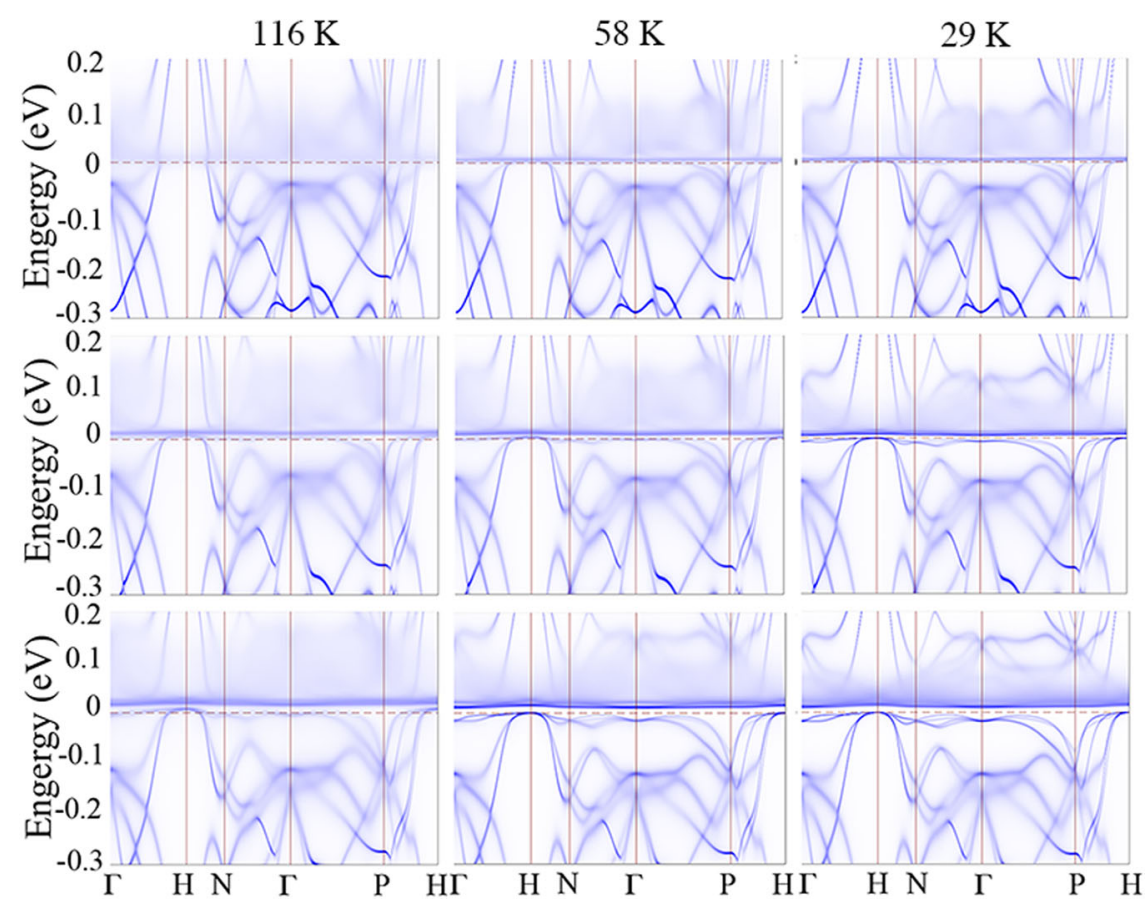

Fig. 1 Momentum-resolved spectral function of $\mathrm{Ce}_{3} \mathrm{Pd}_{3} \mathrm{Bi}_{4}$ from $\mathrm{DFT}+\mathrm{DMFT}$ at 29,58 , and $116 \mathrm{~K}$ for various cell volumes respectively. The panels from top to bottom are $98 \%, 94 \%$, and $90 \%$ compressed $V_{0}$, respectively. From the top-left panel to the right-bottom panel, an energy gap around the Fermi level gradually opens under the lattice shrinking and upon cooling.

and $98 \% V_{0}$ are shown in Fig. 1 , respectively. $V_{0}$ is the experimental unit cell volume at ambient pressure. A gradual change of spectra function from incoherent to coherent behavior near the Fermi level is evident with decreasing temperature and increasing pressure (from the top-left to the right-bottom panel in Fig. 1). For the $98 \%$ compressed Pd-compound (the upper panels in Fig. 1), the spectra functions unequivocally display a blurred region in a wide temperature range from $116 \mathrm{~K}$ down to $29 \mathrm{~K}$, indicative of the incoherent scattering at higher temperatures (see Supplementary Note 1 for details). Increasing the pressure $\left(94 \% V_{0}\right.$ compound), the spectra become sharper near the Fermi level. In particular, a small energy gap appears in the very vicinity of $E_{\mathrm{F}}$ at the temperature of $29 \mathrm{~K}$. For the most compressed compound $\left(90 \% V_{0}\right)$ considered in this work, the energy gap can be identified at even higher temperature, implying the external pressure has driven the system away from the metallicity. It is worth noting that the spectra of $90 \%$ compressed $\mathrm{Ce}_{3} \mathrm{Pd}_{3} \mathrm{Bi}_{4}$ compound at $29 \mathrm{~K}$ resembles that of $\mathrm{Ce}_{3} \mathrm{Pt}_{3} \mathrm{Bi}_{4}$ at $18 \mathrm{~K}^{19}$, suggesting similar effect of external pressure and isoelectronic substitution by $\mathrm{Pt}$, in agreement with the previous study ${ }^{23}$.

To obtain insights into the formation of energy gap upon reducing temperature and increasing external pressure, the hybridization function and integrated spectra density were illustrated in Fig. 2. In general, the intensities of hybridization function (both the real and imaginary part) are gradually enhanced as the cell volume decreases. Simultaneously, the peak position is shifted away from the Fermi level, corresponding to the transfer of spectral weight from $E_{\mathrm{F}}$ to the gap edges. This is the typical behavior of the charge gap formation due to the hybridization between the f-electron and conduction electrons ${ }^{10,24}$. The change in hybridization function is consistent with the integrated spectra density change as shown in Fig. $2 d-f$. The integrated spectra density at $E_{\mathrm{F}}$ initially decreases under the pressure, then begins to show a small dip for $94 \%$ compressed $\mathrm{Pd}-$ compound, and finally forms a broad U-shaped gap for $90 \%$ compressed Pd-compound at $29 \mathrm{~K}$. Such pressure-induced behavior, however, is less obvious at $116 \mathrm{~K}$ due to the collapse of energy gap at high temperature. If we evaluate the gap size $\Delta_{0}$, as illustrated in the insets of Fig. 2, by the energy interval from the half-height of the maximal intensity at the gap edge below $E_{\mathrm{F}}$ to the minimum intensity value inside the gap, $\Delta_{0}$ can be well fitted with a linear function to external pressure (cell volume) at both 29 and $58 \mathrm{~K}$. Similar linear pressure dependence of energy gap was previously reported in $\mathrm{Ce}_{3} \mathrm{Pt}_{3} \mathrm{Bi}_{4}{ }^{12}$. In addition, for a specific pressure (volume), $\Delta_{0}$ is also smoothly dependent on the temperature. Hence the system continuously goes through a crossover from a metallic regime to an insulating regime upon cell volume compressing at finite temperature.

Having qualitatively established the presence of metal-insulator crossover below some certain characteristic temperature at various compressed cell volumes, we now address the question whether the ground state $(T \rightarrow 0)$ of $\mathrm{Ce}_{3} \mathrm{Pd}_{3} \mathrm{Bi}_{4}$ at ambient conditions is characterized by the Kondo insulating or f-electron-incoherent metallic states. As mentioned above, the spectral intensity at $E_{\mathrm{F}}$ gradually reduces upon cooling, resulting in a red-shift of valence bands edge $E_{\mathrm{v}}$ due to the formation of energy gap. Once the top of the valence band shifts below $E_{\mathrm{F}}$, the Fermi level $E_{\mathrm{F}}$ is pinned inside the gap, leading the system towards the insulating regime when $T>$ $0 \mathrm{~K}$. Therefore, a characteristic temperature $T_{1}$ can be defined when $E_{\mathrm{v}}$ coincides with $E_{\mathrm{F}}$. Below $T_{1}$, the electronic states around the Fermi level shall eventually become coherent, and a true gap shall appear at sufficiently low temperature. Then we examine the shift of valence bands edge $E_{\mathrm{v}}$ relative to the Fermi level for various compressed cell volumes in the temperature range from 18 to $116 \mathrm{~K}$. The calculated results are shown in Fig. 3a-e. By extrapolating to zero temperature, we find the valence band edge cuts the Fermi level at $T_{1}$ of $6.4,14.5,26.7,32.8$, and $44.8 \mathrm{~K}$ for $98 \%, 96 \%, 94 \%, 92 \%$, and $90 \% V_{0}$, respectively. It indicates that the ground states are insulating at $0 \mathrm{~K}$ for the corresponding cell volume. In Fig. 3f, we show the varying of $T_{1}$ as a function of cell volume. The $T_{1}$ linearly decreases as the lattice expands, which is in line with the aforementioned linear suppression of energy gap under small increasing of cell volume ${ }^{21}$. The temperature scale $T_{1}$ eventually disappears at $99.2 \% V_{0}$ (corresponding to the hydrostatic pressure of 1.1 $\mathrm{GPa}$, see Supplementary Note 2), implying that the insulating 
$29 \mathrm{~K}$
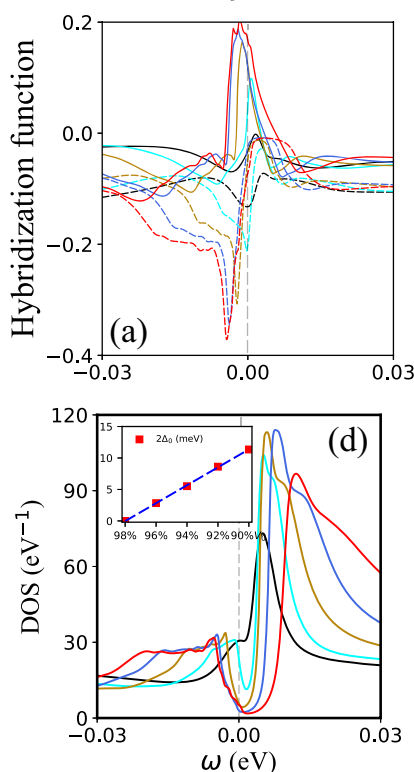

$58 \mathrm{~K}$
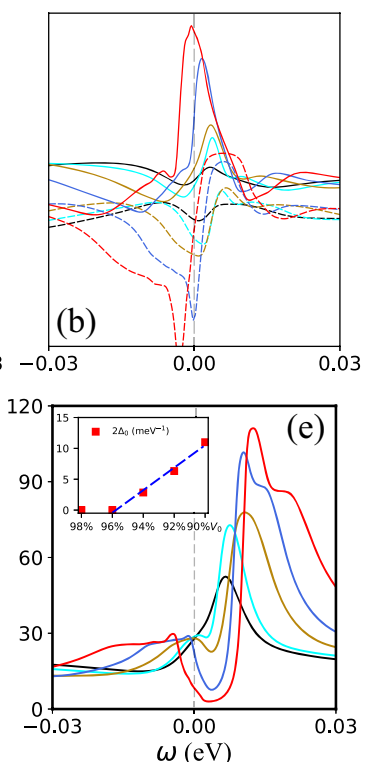

$116 \mathrm{~K}$
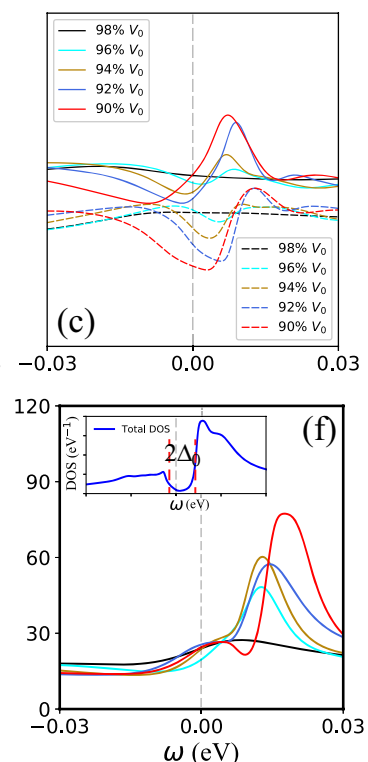

Fig. 2 Pressure dependence of hybridization function and local spectra density. a-c The real (solid lines) and imaginary (dashed lines) part of the hybridization function. $\mathbf{d}$-f the integrated spectra density at 29,58 , and $116 \mathrm{~K}$, for $98 \%, 96 \%, 94 \%$, $92 \%$, and $90 \%$ compressed cell volume, respectively. The magnitude of the energy gap is plotted as the function of cell volume shown in the inset of (d, e). The gap size is evaluated from the half-height of the maximal intensity at the gap edge below $E_{\mathrm{F}}$ to the minimal intensity value inside the gap as schematically illustrated in the inset of (f). The black dashed line in the inset of (f) marks the valence bands edge $E_{\mathrm{v}}$.
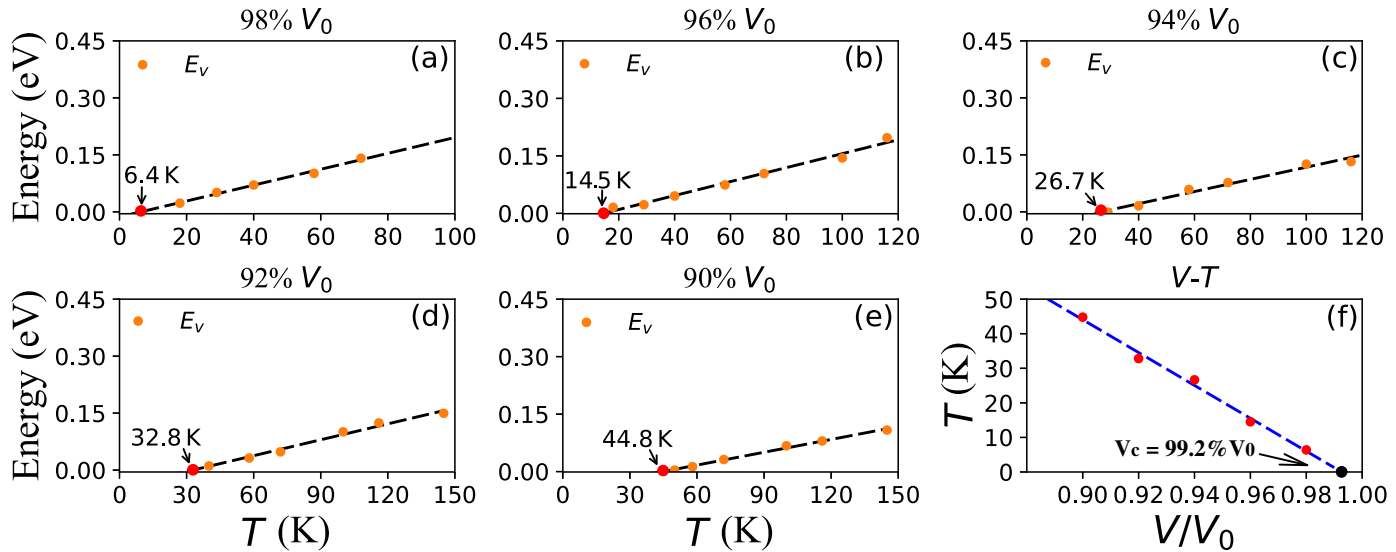

Fig. 3 Temperature and pressure dependence of valence band edge. a-e Temperature evolution of valence band edge $E_{\mathrm{v}}$ at $98 \%, 96 \%, 94 \%$, $92 \%$, and $90 \%$ of the equilibrium volume $V_{0}$, respectively. The tops of the valence bands relative to the Fermi level are marked as blue (orange) dots. The gray dashed lines are linear fittings to $E_{\mathrm{v}}$. The black arrow indicates the extrapolated critical temperatures $T_{1}$ (marked as red dots) when the $E_{\mathrm{v}}$ cuts the Fermi level. f Pressure evolution of $T_{1}$. The red dots mark $T_{1}$ from (a-e). The blue dashed line is the linear extrapolation to zero temperature.

phase cannot be stabilized at $0 \mathrm{~K}$ when the cell volume is larger than this value.

\section{Magnetic susceptibility}

To further verify this, we have also calculated the local magnetic susceptibility $X_{\text {loc }}$ for different cell volumes. The results are shown in Fig. 4 (also see Supplementary Note 3). Upon lattice shrinking, the Curie-like susceptibility gradually turns to be Pauli-like with much weaker temperature dependence, indicating the reduction of the local moment due to the Kondo screening. The quantity $T^{*}$ evaluated from the maximal of $X_{\mathrm{loc}}$ is the characteristic temperature below which the local moment of $f$-electron and the conduction electron form an entangle state, e.g., the Kondo singlet, and the system starts entering into the coherent regime with the Fermi-liquid behavior. As the cell volume decreases, $T^{*}$ gradually increases, signaling the tendency of $f$-electron coherence from a low-pressure phase to a high-pressure phase ${ }^{25,26}$. In the inset of Fig. 4, we show $T^{*}$ as a function of cell volume, which is qualitatively similar to the previous experimental observations ${ }^{18}$. Intriguingly, $T^{*}$ also approaches to zero at $\sim 99 \% V_{0}$, concomitant with the disappearance of the delocalization-localization crossover temperature, $T_{1}$, at the same cell volume. This strongly suggests that, under the ambient pressure, the Ce-4f-electrons in $\mathrm{Ce}_{3} \mathrm{Pd}_{3} \mathrm{Bi}_{4}$ compound remains localized even at zero temperature.

\section{The upper limit of crossover temperature}

Furthermore, we can also estimate another characteristic temperature $T_{2}$ at which the energy gap $\Delta_{0}$ begins to develop around $E_{\mathrm{F}}$. It is worth noting that the presence of such a gap at 


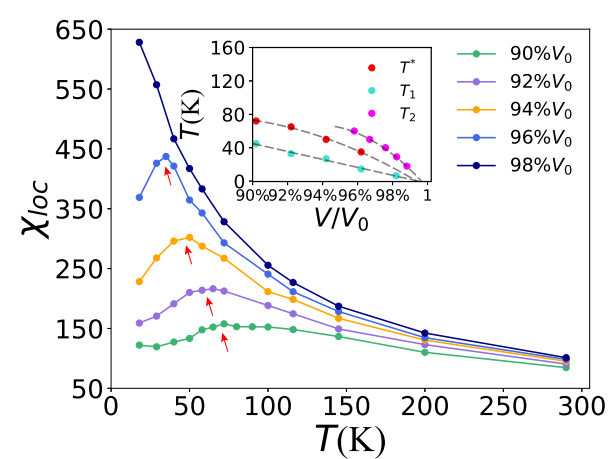

Fig. 4 Temperature dependence of local magnetic susceptibility Xloc for various compressed cell volumes. The inset shows the temperature versus pressure phase diagram. The characteristic temperature $T^{*}$ (marked with the red dots) associated with the formation of Kondo singlet, is corresponding to the maximums of the local magnetic susceptibility marked with red arrows in the main figure. Correspondingly, we show the $1 / X_{\text {loc }}$ in Supplementary Note 3. In comparison, the pressure evolution of $T_{1}$ and $T_{2}$ from Fig. $3 \mathrm{f}$ and Supplementary Fig. 5a are illustrated in the inset as well. The dashed line indicates the gradual suppression trend of $T^{*}$ upon lattice compression. Note that for $98 \% V_{0} \mathrm{Pd}$-compound, no maximum of Xloc can be identified down to $18 \mathrm{~K}$.

finite temperatures does not guarantee an insulating phase because the system is susceptible to the incoherent in-gap state and the alignment of the Fermi level due to the thermal effect. Therefore, it poses an upper limit of corresponding to $f$-electron localization-delocalization crossover temperature. However, as the temperature approaches 0 , the thermal effect diminishes, and the extrapolation also approaches $P_{\mathrm{c}}$ at $1.1 \mathrm{GPa}$ (Fig. 4 inset, as well as Supplementary Note 4).

\section{DISCUSSION}

The following remarks and discussions are in order: first, as we have stated previously, the effect of external pressure is similar to the isoelectronic substitution by $\mathrm{Pt}$. In fact, previous DFT study has shown that the radial extent of $\mathrm{Pd}(\mathrm{Pt})-4 d(5 d)$ shall affect the hybridization strength ${ }^{22}$. Therefore, the localizationdelocalization transition may also be realized with Ptsubstitution. Second, we have observed scaling behavior in the Kondo insulating regime between unrenormalized energy gap $\Delta_{0} / Z$ and the maximum of hybridization function $\operatorname{Im}\left[\Gamma_{\max }(\omega)\right]$, defined as the peak value of imaginary part of hybridization function near the Fermi level, which suggests a critical hybridization strength of $\operatorname{Im}\left[\Gamma_{\max }(\omega)\right] \approx-0.15 \mathrm{eV}$. This is also consistent with the critical $P_{c}$ around $1 \mathrm{GPa}$ (see Supplementary Note 4 for details). Thirdly, it is informative to mention that the Hubbard $U$ has a moderate influence on the critical value of the gap as well. Since the larger value of $U$ yields more localized Ce-4f moment, which is in turn harmful to a finite magnitude of energy gap, a larger/smaller $U$ would result in a slightly larger/smaller critical pressure $P_{c}$. Nevertheless, the controversial experimental results ${ }^{18,21}$ indicate the close proximity of the ambient system close to the critical pressure, which is arguably accessible with small pressure. It is also worth noting that a similar effect of $f$-electrons localization-delocalization can also be achieved by applying magnetic field in addition to external pressure, which may serve as an explanation for the observed simultaneous vanishing of $T^{*}$ and $T_{1}$ at critical magnetic field $B_{\mathrm{c}} \approx 11 \mathrm{Tesla}^{21}$. Finally, at the Kondo insulating regime, we have employed topological Hamiltonian $^{27,28}$ and Wilson loop method ${ }^{29}$ to show that the system is topologically trivial (see Supplementary Note 5). Since at the $f$-electron-incoherent metallic state, the system is topological nodal-line semimetals as suggested in ref. ${ }^{19}$, it can be inferred that the $f$-electron localization-delocalization transition is accompanied by a topological phase transition as well.

In conclusion, we have performed a comprehensive DFT + DMFT study of the pressure-/temperature-dependent electronic structure of $\mathrm{Ce}_{3} \mathrm{Pd}_{3} \mathrm{Bi}_{4}$. At ambient pressure, the ground state of $\mathrm{Ce}_{3} \mathrm{Pd}_{3} \mathrm{Bi}_{4}$ at $\mathrm{OK}$ is found to be metallic due to insufficient hybridization between the $\mathrm{Ce}-4 \mathrm{f}$ electron and conduction electrons. As the pressure increases, the hybridization enhances, and a metal-insulator transition occurs $\sim 99 \% V_{0}$. Using the topological Hamiltonian and Wilson loop method, the pressurized insulating phase is found to be a topologically trivial Kondo insulator. This leads to the observation that the metal-insulator transition is accompanied by a topological transition as well. Our results suggest that a possible topological quantum critical point may be achieved by applying pressure in $\mathrm{Ce}_{3} \mathrm{Pd}_{3} \mathrm{Bi}_{4}$.

\section{METHODS}

To describe the electron correlation and magnetic fluctuation of $\mathrm{Ce}-$ compounds, the combination of density function theory (DFT) and DMFT $^{30,31}$ was employed. The DFT part was performed with the fullpotential linear augmented plane-wave method implemented in the Wien2k package ${ }^{32}$ with generalized gradient approximation ${ }^{33}$. The continuous-time Monte Carlo method (CTQMC) ${ }^{34}$ was used as the impurity solver in its fully self-consistent version of DFT+DMFT. The Coulomb interaction $U=6.0 \mathrm{eV}$ and Hund's coupling $J=0.7 \mathrm{eV}$ were considered for Ce-4f orbitals (see Supplementary Note 1 for details). Since the simulated pressures are rather small, the internal atomic positions were kept the same as those under the ambient condition when changing the lattice constant to simulate the external pressure, The topological Hamiltonian $h_{\mathrm{t}}(\mathbf{k})=h_{0}(\mathbf{k})+\Sigma(\omega=0)$ can be obtained after the self-consistent DFT + DMFT calculations. The zerofrequency self-energy $\Sigma(0)$ was also extrapolated to zero temperature. Both $h_{0}(\mathbf{k})$ and $\Sigma(0)$ are symmetrized with the WannSymm code ${ }^{35}$.

\section{DATA AVAILABILITY}

The data that support the findings of this study are available from the corresponding author upon reasonable request.

\section{CODE AVAILABILITY}

The latest WannSymm code is available on http://github.com/ccao/WannSymm.

Received: 17 September 2021; Accepted: 13 January 2022; Published online: 03 February 2022

\section{REFERENCES}

1. Doniach, S. The Kondo lattice and weak antiferromagnetism. Physica $B+C$ 91, 231-234 (1977).

2. Sachdev, S. Quantum magnetism and criticality. Nat. Phys. 4, 173-185 (2008).

3. Löhneysen, H. V., Rosch, A., Vojta, M. \& Wölfle, P. Fermi-liquid instabilities at magnetic quantum phase transitions. Rev. Mod. Phys. 79, 1015-1075 (2007).

4. Gegenwart, P., Si, Q. \& Steglich, F. Quantum criticality in heavy-fermion metals. Nat. Phys. 4, 186-197 (2008).

5. Isobe, H., Yang, B.-J., Chubukov, A., Schmalian, J. \& Nagaosa, N. Emergent nonFermi-liquid at the quantum critical point of a topological phase transition in two dimensions. Phys. Rev. Lett. 116, 076803 (2016).

6. Wang, J.-R., Liu, G.-Z. \& Zhang, C.-J. Topological quantum critical point in a tripleWeyl semimetal: Non-Fermi-liquid behavior and instabilities. Phys. Rev. B 99, 195119 (2019).

7. Assaad, F. F. \& Herbut, I. F. Pinning the order: The nature of quantum criticality in the Hubbard model on honeycomb lattice. Phys. Rev. X 3, 031010 (2013).

8. Bi, Z. \& Senthil, T. Adventure in topological phase transitions in 3+1-d: NonAbelian deconfined quantum criticalities and a possible duality. Phys. Rev. X 9, 021034 (2019). 
9. Jaime, M. et al. Closing the spin gap in the Kondo insulator $\mathrm{Ce}_{3} \mathrm{Bi}_{4} \mathrm{Pt}_{3}$. Nature 405, 160-163 (2000)

10. Bucher, B., Schlesinger, Z., Canfield, P. C. \& Fisk, Z. Kondo coupling induced charge gap in $\mathrm{Ce}_{3} \mathrm{Bi}_{4} \mathrm{Pt}_{3}$. Phys. Rev. Lett. 72, 522-525 (1994).

11. Hundley, M. F., Canfield, P. C., Thompson, J. D., Fisk, Z. \& Lawrence, J. M. Hybridization gap in $\mathrm{Ce}_{3} \mathrm{Bi}_{4} \mathrm{Pt}_{3}$. Phys. Rev. B 42, 6842-6845 (1990).

12. Cooley, J. C., Aronson, M. C. \& Canfield, P. C. High pressures and the Kondo gap in $\mathrm{Ce}_{3} \mathrm{Bi}_{4} \mathrm{Pt}_{3}$ s. Phys. Rev. B 55, 7533-7538 (1997).

13. Takegahara, K., Harima, H., Kaneta, Y. \& Yanase, A. Electronic band structures of $\mathrm{Ce}_{3} \mathrm{Pt}_{3} \mathrm{Sb}_{4}$ and $\mathrm{Ce}_{3} \mathrm{Pt}_{3} \mathrm{Bi}_{4}$. J. Phys. Soc. Jpn 62, 2103-2111 (1993).

14. Dzero, M., Sun, K., Galitski, V. \& Coleman, P. Topological Kondo insulators. Phys. Rev. Lett. 104, 106408 (2010).

15. Chang, P.-Y., Erten, O. \& Coleman, P. Möbius Kondo insulators. Nat. Phys. 13 794-798 (2017).

16. Alexandrov, V., Dzero, M. \& Coleman, P. Cubic topological Kondo insulators. Phys. Rev. Lett. 111, 226403 (2013).

17. Lai, H.-H., Grefe, S. E., Paschen, S. \& Si, Q. Weyl-Kondo semimetal in heavyfermion systems. Proc. Natl Acad. Sci. USA 115, 93-97 (2018).

18. Dzsaber, S. et al. Kondo insulator to semimetal transformation tuned by spin-orbit coupling. Phys. Rev. Lett. 118, 246601 (2017).

19. Cao, C., Zhi, G.-X. \& Zhu, J.-X. From trivial Kondo insulator $\mathrm{Ce}_{3} \mathrm{Pt}_{3} \mathrm{Bi}_{4}$ to topological nodal-line semimetal $\mathrm{Ce}_{3} \mathrm{Pd}_{3} \mathrm{Bi}_{4}$. Phys. Rev. Lett. 124, 166403 (2020).

20. Dzsaber, S. et al. Giant spontaneous Hall effect in a nonmagnetic Weyl-Kondo semimetal. Proc. Natl Acad. Sci. USA 118, e2013386118 (2021).

21. Kushwaha, S. K. et al. Magnetic field-tuned Fermi liquid in a Kondo insulator. Nat. Commun. 10, 5487 (2019).

22. Tomczak, J. M. Isoelectronic tuning of heavy fermion systems: proposal to synthesize $\mathrm{Ce}_{3} \mathrm{Sb}_{4} \mathrm{Pd}_{3}$. Phys. Rev. B 101, 035116 (2020).

23. Pickem, M., Maggio, E. \& Tomczak, J. M. Resistivity saturation in Kondo insulators. Commun. Phys. 4, 226 (2021).

24. Takeda, Y. et al. High-resolution photoemission study of the temperaturedependent $c-f$ hybridization gap in the Kondo semiconductor $\mathrm{YbB}_{12}$. Phys. Rev. B 73, 033202 (2006).

25. Marianetti, C. A., Haule, K., Kotliar, G. \& Fluss, M. J. Electronic coherence in $\delta$-Pu: a dynamical mean-field theory study. Phys. Rev. Lett. 101, 056403 (2008)

26. Chakrabarti, B., Pezzoli, M. E., Sordi, G., Haule, K. \& Kotliar, G. $a-\gamma$ transition in cerium: magnetic form factor and dynamic magnetic susceptibility in dynamical mean-field theory. Phys. Rev. B 89, 125113 (2014).

27. Wang, Z. \& Zhang, S.-C. Simplified topological invariants for interacting insulators. Phys. Rev. X 2, 031008 (2012).

28. Wang, Z. \& Yan, B. Topological Hamiltonian as an exact tool for topological invariants. J. Phys. Conden. Matter 25, 155601 (2013).

29. Yu, R., Qi, X. L., Bernevig, A., Fang, Z. \& Dai, X. Equivalent expression of $Z_{2}$ topological invariant for band insulators using the non-Abelian Berry connection. Phys. Rev. B 84, 075119 (2011).

30. Georges, A., Kotliar, G., Krauth, W. \& Rozenberg, M. J. Dynamical mean-field theory of strongly correlated fermion systems and the limit of infinite dimensions. Rev. Mod. Phys. 68, 13-125 (1996).

31. Kotliar, G. et al. Electronic structure calculations with dynamical mean-field theory. Rev. Mod. Phys. 78, 865-951 (2006).

32. Schwarz, K., Blaha, P. \& Madsen, G. K. Electronic structure calculations of solids using the Wien2k package for material sciences. Comput. Phys. Commun. 147, 71-76 (2002).

33. Perdew, J. P., Burke, K. \& Ernzerhof, M. Generalized gradient approximation made simple. Phys. Rev. Lett. 77, 3865-3868 (1996).

34. Haule, K. Quantum Monte Carlo impurity solver for cluster dynamical mean-field theory and electronic structure calculations with adjustable cluster base. Phys. Rev. B 75, 155113 (2007).
35. Zhi, G.-X., Xu, C., Wu, S.-Q., Ning, F. \& Cao, C. Wannsymm: a symmetry analysis code for wannier orbitals. Comput. Phys. Commun. 271, 108196 (2022).

\section{ACKNOWLEDGEMENTS}

The authors thank Jianhui Dai, Priscila Rosa, Qimiao Si, Huiqiu Yuan, and Wei Zhu for the discussions. The work at Los Alamos was carried out under the auspices of the U.S. Department of Energy (DOE) National Nuclear Security Administration under Contract No. 89233218CNA000001. It was supported by Pioneer and Leading Goose R\&D Program of Zhejiang 2022SDXHDX0005 (C.C), National Key R\&D Program of the MOST of China 2017 YFA0303100 $\quad$ (C.C.), NSFC 11874137 (C.C. and C.X.), and the LANL LDRD Program (J.-X.Z.). J.-X.Z. was also supported by the Center for Integrated Nanotechnologies, a U.S. DOE BES user facility. The calculations were performed on the High-Performance Computing Center at Hangzhou Norma University, High-Performance Computing Cluster of Center of Correlated Matters at Zhejiang University, and Beijing Super-Computing Center.

\section{AUTHOR CONTRIBUTIONS}

C.C. and J.-X.Z. initiated this work; C.X. and C.C. performed the calculations and were responsible for the data analysis; J.-X.Z. participated in the interpretation of numerical results. All authors contributed to the manuscript.

\section{COMPETING INTERESTS}

The authors declare no competing interests.

\section{ADDITIONAL INFORMATION}

Supplementary information The online version contains supplementary material available at https://doi.org/10.1038/s41535-022-00427-9.

Correspondence and requests for materials should be addressed to Chao Cao or Jian-Xin Zhu.

Reprints and permission information is available at http://www.nature.com/ reprints

Publisher's note Springer Nature remains neutral with regard to jurisdictional claims in published maps and institutional affiliations.

(i) Open Access This article is licensed under a Creative Commons Attribution 4.0 International License, which permits use, sharing, adaptation, distribution and reproduction in any medium or format, as long as you give appropriate credit to the original author(s) and the source, provide a link to the Creative Commons license, and indicate if changes were made. The images or other third party material in this article are included in the article's Creative Commons license, unless indicated otherwise in a credit line to the material. If material is not included in the article's Creative Commons license and your intended use is not permitted by statutory regulation or exceeds the permitted use, you will need to obtain permission directly from the copyright holder. To view a copy of this license, visit http://creativecommons. org/licenses/by/4.0/.

(c) The Author(s) 2022 